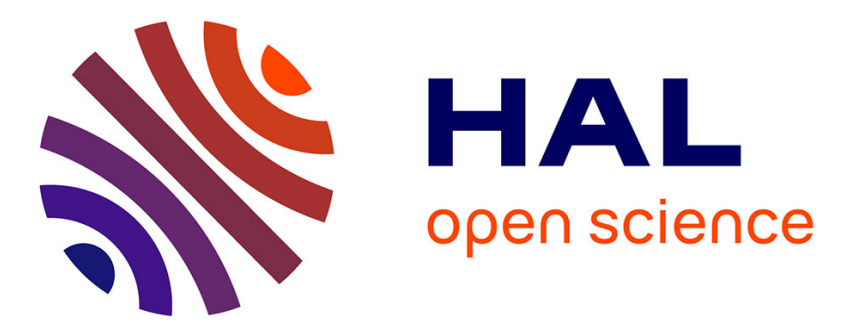

\title{
Performance Analysis of Multistream Receive Spatial Modulation in the MIMO Broadcast Channel
}

\author{
Athanasios Stavridis, Marco Di Renzo, Harald Haas
}

\section{To cite this version:}

Athanasios Stavridis, Marco Di Renzo, Harald Haas. Performance Analysis of Multistream Receive Spatial Modulation in the MIMO Broadcast Channel. IEEE Transactions on Wireless Communications, 2016, 15 (3), pp.1808 - 1820. 10.1109/TWC.2015.2496597 . hal-01880186

\section{HAL Id: hal-01880186 https://hal.science/hal-01880186}

Submitted on 7 Jul 2020

HAL is a multi-disciplinary open access archive for the deposit and dissemination of scientific research documents, whether they are published or not. The documents may come from teaching and research institutions in France or abroad, or from public or private research centers.
L'archive ouverte pluridisciplinaire HAL, est destinée au dépôt et à la diffusion de documents scientifiques de niveau recherche, publiés ou non, émanant des établissements d'enseignement et de recherche français ou étrangers, des laboratoires publics ou privés. 


\title{
Performance Analysis of Multi-Stream Receive Spatial Modulation in the MIMO Broadcast Channel
}

\author{
Athanasios Stavridis, Marco Di Renzo, Senior Member, IEEE, and Harald Haas, Member, IEEE
}

\begin{abstract}
In this paper, Multi-Stream Receive-Spatial Modulation (MSR-SM) for application to the Multiple-Input MultipleOutput (MIMO) broadcast channel is introduced and studied. MSR-SM is a closed-loop transmission scheme, which applies the concept of multi-stream space modulation at the receiver side. An accurate mathematical framework for the evaluation of the Bit Error Rate (BER) is proposed. In addition, the diversity order and coding gain of the new architecture are derived. Note that the proposed analytical framework takes into account both the smallscale fading and the system topology, and is directly applicable to the conventional MIMO broadcast channel. Compared with the state-of-the-art MIMO transmission in the broadcast channel, it is mathematically shown that MSR-SM achieves the same diversity order and a better coding gain, in the high Signal-toNoise Ratio (SNR) regime. Finally, the proposed mathematical framework and the new findings are validated via Monte Carlo simulation results.
\end{abstract}

\section{INTRODUCTION}

$\mathbf{M}$ ULTI-ANTENNA communication has been considered as a promising technique for achieving high data rates without requiring additional radio resources $[1,2]$. However, due to the deployment of multiple antennas, the complexity of the transceiver could become prohibitively high. A MultipleInput Multiple-Output (MIMO) scheme which promotes a low complexity implementation is Spatial Modulation (SM) [3-8]. Due to its operating mechanism, SM requires a single Radio Frequency (RF) front-end at the transmitter [3]. This is shown to offer significant energy gains compared with conventional MIMO techniques $[9,10]$. In addition, at the receiver side, a low complexity (single stream) Maximum Likelihood (ML) detector is deployed [8]. Despite the adoption of a single stream detector, SM is able to obtain a multiplexing gain.

Inspired by the potential of SM, several authors have extended the concept of SM in different communication scenarios [11-23]. For example, Space Shift Keying (SSK) is a low complexity and low rate variant of SM [11]. Furthermore, Space Time Shift Keying (STSK) is a SM-based scheme which extends the concept of SM in the time domain [24]. The first real system implementation of SM has recently

This work was supported in part by the European Commission through the FP7-PEOPLE ITN-GREENET project under Grant 264759. Professor Harald Haas acknowledges support from the EPSRC under Established Career Fellowship grant EP/K008757/1. This paper was presented in part at the 2015 IEEE Global Communications Conference (GLOBECOM), San Diego, California, USA, December 2015.

A. Stavridis and H. Haas are with the Li-Fi Research and Development Centre, Institute for Digital Communications, School of Engineering, The University of Edinburgh, Edinburgh, UK. E-mail: \{a.stavridis, h.haas\}@ed.ac.uk.

M. Di Renzo is with the Laboratoire des Signaux et Systèmes, CNRS, CentraleSupélec, Univ Paris Sud, Université Paris-Saclay, 3 rue Joliot Curie, Plateau du Moulon, 91192, Gif-sur-Yvette, France. E-mail: marco.direnzo@12s.centralesupelec.fr. been reported in [25]. The performance of SM under real channel measurements is discussed in $[26,27]$. A complete introduction on SM is provided in [3].

\section{A. Related Work and Motivation}

Similar to the concept of conventional SM, the author of [28] proposes a point-to-point closed-loop MIMO scheme that applies the principle of SM at the receiver side. In particular, using MIMO linear precoding, the reciprocal of SM, called Receive-Spatial Modulation (R-SM), is obtained. The extension of R-SM to a scheme which spatially modulates multiple parallel symbol streams to the indices of multiple receiving antennas is conducted in $[29,30]$. In the present paper, the term of Multi-Stream Receive-Spatial Modulation (MSR-SM) is used for this scheme. In addition, the performance of R-SM in different application scenarios is studied in [31-36].

As discussed in [3], there is a wide range of spatially modulated architectures for point-to-point communication. However, is it possible for SM to be incorporated in a Multi-User $(M U)$ scenario? Indeed, just like any other MIMO physical layer technique, SM and its variants can be combined with a multiple access scheme such as Time Division Multiple Access (TDMA), Frequency Division Multiple Access (FDMA), or Orthogonal Frequency-Division Multiple Access (OFDMA) in order to form a MU system.

A new trend in MIMO communication promotes systems where multiple users are aggressively allocated in the same time and frequency resources. Usually, this is accomplished via Space Division Multiple Access (SDMA) techniques [37,38]. However, the design of a SDMA-based spatially modulated scheme for the downlink is a challenging task. Due to the activation of a single transmit antenna (or a subset of the available transmit antennas) and the way that information is conveyed, the design of interference reduction, elimination, or manipulation techniques becomes difficult. However, the authors of $[39,40]$ managed to incorporate SM in the MIMO broadcast channel. In these papers, non-interfering SM-based communication is established via the use of a linear precoding matrix which is based on the Zero Forcing (ZF) principle.

\section{B. Contributions and Outcomes}

Against this background, the present paper aims to incorporate MSR-SM in the MIMO broadcast channel. More specifically, a new SDMA architecture based on the concept of MSR-SM is proposed. In addition, an accurate mathematical framework for computing the Average Bit Error Probability (ABEP), the diversity order, and the coding gain is introduced. 
The proposed transmission scheme and framework, in particular, are based on the following assumptions: i) the wireless channel follows a Rayleigh distribution; ii) Perfect-Channel State Information at the Transmitter (P-CSIT) is assumed; iii) the proposed precoder is designed based on the $\mathrm{ZF}$ principle; and iv) the effect of system topology is duly taken into account. Based on the proposed mathematical framework, it is proved that the proposed scheme is capable of outperforming the conventional MIMO broadcast channel. In particular, the proposed scheme provides the same diversity order as stateof-the-art MIMO scheme but a better coding gain in the high Signal-to-Noise Ratio (SNR) regime.

As far as the novelty of the proposed mathematical framework is concerned, to the best of the authors' knowledge, the analysis of the diversity order and of the coding gain of MSR-SM is not available in the literature. In the present paper, we prove, for the first time, that the conventional MIMO broadcast channel and MU MSR-SM achieve the same diversity order if they both rely on ZF precoding. In addition, the proposed approach is directly applicable to pointto-point single user scenarios. As far as this latter scenario is concerned, in particular, it is worth mentioning that the ABEP of MSR-SM for point-to-point single user transmission has recently been studied in $[29,30]$. However, several important differences exist between the framework available in $[29,30]$ and that proposed in the present paper. The study presented in [29] does not take into account the statistical description of the received signal. The ABEP computed in [30] is applicable to MSR-SM in the presence of a suboptimal detector, which decouples the detection process. In contrast, the analysis presented in Sections IV and $\mathrm{V}$ is different for the following reasons: i) the statistical description of the received signal is considered; ii) the system topology that is inherent in MU setups is taken into account; and iii) the detection process is based on the ML principle which imposes some additional mathematical difficulties. Finally, since the conventional Spatial MultipleXing (SMX) MIMO architecture with ZF precoding is a special case of MSR-SM, the proposed framework can be applied to this setup as well and can be used for a simple comparison between the two architectures, as better discussed in the sequel.

The rest of the present paper is organized as follows. In Section II, the system model is introduced. In Section IV, the ABEP of each user and of the whole system is computed. In Section V, the diversity order and coding gain of MSR-SM in the MIMO broadcast channel are analyzed. In Section VI, the proposed MSR-SM MIMO architecture is compared against the corresponding conventional MIMO broadcast channel and some numerical results that validate our theoretical findings are illustrated. Finally, Section VII concludes this paper.

Notation: Lowercase bold letters denote vectors and uppercase bolt letters denote matrices. $(\cdot)^{T},(\cdot)^{H}, \operatorname{tr}(\cdot)$ and $\mathbf{A}^{1 / 2}$ denote transpose, Hermitian transpose, matrix trace and the square root of $\mathbf{A}$, respectively. The Kronecker product is denoted as $\otimes$. $\|\cdot\|_{2}$ represents the Euclidean norm, while $\|\cdot\|_{F}$ is the Frobenius norm. A diagonal matrix, whose main diagonal includes the elements $a_{1}, \cdots, a_{n}$, is denoted as $\operatorname{diag}\left(a_{1}, \ldots, a_{n}\right)$. E[·] denotes the mean value of a RV. A complex Gaussian distribution with mean $m$ and variance $\sigma_{C}^{2}$ is represented as $\mathcal{C N}\left(m, \sigma_{C}^{2}\right)$, where its real and imaginary part are independent and identically distributed (i.i.d.) Gaussian RV with distribution $\mathcal{N}\left(m, \frac{\sigma_{C}^{2}}{2}\right) . \operatorname{Re}\{\cdot\}$ denotes the real part of a complex number or matrix.

\section{SySTEM MODEL}

An uncoded Multi-User Multiple-Input Multiple-Output (MU-MIMO) system that comprises a multi-antenna Base Station (BS) and $N_{u}$ remotely distributed multi-antenna users is considered. The BS is equipped with $N_{t}$ antennas and each user possesses $N_{r}$ antennas. Since the transmitter is a BS, it is realistic to consider the assumption that $N_{t} \geq N_{u} N_{r}$. In addition, the wireless channel between the BS and every user is assumed to be frequency flat and quasi-static. Finally, P-CSIT is considered, which can be obtained by using either the channel reciprocity or fast and error free links from the users $^{1}$.

Provided the availability of P-CSIT, the transmitter is able to use linear precoding. By interpreting the $N_{t}$ transmit antennas and the $B=N_{u} N_{r}$ receive antennas as a $N_{t} \times B$ MIMO system, the baseband equation of such a system is expressed as:

$$
\mathbf{y}=\breve{\mathbf{H}} \breve{\mathbf{P}} \breve{\mathbf{D}} \mathbf{x}+\mathbf{w}
$$

in a matrix form. In (1), $\mathbf{y}=\left[\mathbf{y}_{1}^{T}, \ldots, \mathbf{y}_{N_{u}}^{T}\right]^{T}$ is a $N_{u} N_{r} \times 1$ vector, where $\mathbf{y}_{i}, i=1, \ldots, N_{u}$, denotes the $N_{r} \times 1$ received signal vector at the $i$-th user. The $N_{u} N_{r} \times N_{t}$ matrix, $\breve{\mathbf{H}}=\left[\breve{\mathbf{H}}_{1}^{H}, \ldots, \breve{\mathbf{H}}_{N_{u}}^{H}\right]^{H}$, denotes the wireless channel from the transmitter to all receive antennas. Furthermore, the submatrix $\breve{\mathbf{H}}_{i}, i=1, \ldots, N_{u}$, denotes the wireless channel from the transmitter to the $i$-th user. Due to the spatial distribution of the users inside the geographical area of a cell, each user experiences a different large-scale channel effect. However, given that the receive antennas of each user are collocated, the large-scale channel effect between the transmitter and each receive antenna of a certain user is the same. Therefore, it is assumed that $\breve{\mathbf{H}}_{i}, i=1, \ldots, N_{u}$, has the following distribution: $\breve{\mathbf{H}}_{i} \sim \mathcal{C N}\left(\mathbf{0}, \xi_{i} \mathbf{I}\right)$. Here, the value of $\xi_{i}$ is determined by the transmission distance and the effect of shadowing. In general, the values of $\xi_{i}$ close to zero represent a poor channel condition, while those of $\xi_{i}$ close to one indicate a strong channel condition. It is assumed that there is no channel correlation due to rich scattering. The $N_{t} \times N_{u} N_{r}$ precoding matrix can be formulated as $\breve{\mathbf{P}}=\left[\breve{\mathbf{P}}_{1}, \ldots, \breve{\mathbf{P}}_{N_{u}}\right]$, where, $\breve{\mathbf{P}}_{i}, i=1, \ldots, N_{u}$, corresponds to the precoding matrix of the $i$-th user. In order to ensure that the transmitted power is not amplified by the precoder $\breve{\mathbf{P}}$, a $N_{u} N_{r} \times N_{u} N_{r}$ diagonal normalization matrix $\breve{\mathbf{D}}=\operatorname{diag}\left(\breve{d}_{1}, \ldots, \breve{d}_{N_{u} N_{r}}\right)$ is used. Every element $\breve{d}_{i}, i=1, \ldots, N_{u} N_{r}$, of $\breve{\mathbf{D}}$ is expressed as, $\breve{d}_{i}=\sqrt{1 /\left\|\breve{\mathbf{p}}_{i}\right\|_{2}^{2}}$, where, $\breve{\mathbf{p}}_{i}$ is the $i$-th column of $\breve{\mathbf{P}}$. Thus, every column of the normalized precoding matrix, $\breve{\mathbf{P}}_{\text {norm }}=\breve{\mathbf{P}} \breve{\mathbf{D}}$ has unity power. The normalization matrix $\breve{\mathbf{D}}$

\footnotetext{
${ }^{1}$ In real systems, Channel State Information at the Transmitter (CSIT) is subjected to imperfections. However, the study of the effect of imperfect CSIT is out of the scope of this paper.
} 


$$
\mathbf{x}_{i}=[0, \ldots, 0, \underbrace{s_{1}}_{i_{1} \text {-th position }}, 0, \ldots, 0, \underbrace{s_{i}}_{i_{k} \text {-th position }}, 0, \ldots, 0, \underbrace{s_{N_{s}}}_{i_{N_{s}} \text {-th position }} 0 \ldots, 0]^{T}
$$

can be expressed in the following block diagonal matrix form, $\breve{\mathbf{D}}=\operatorname{diag}\left(\breve{\mathbf{D}}_{1}, \ldots, \breve{\mathbf{D}}_{N_{u}}\right)$. Here, $\breve{\mathbf{D}}_{i}, i=1, \ldots, N_{u}$, is the $N_{r} \times N_{r}$ diagonal normalization matrix of the corresponding precoding matrix $\breve{\mathbf{P}}_{i}$. The collective signal vector at the transmitter is denoted as, $\mathbf{x}=\left[\mathbf{x}_{1}^{T}, \ldots, \mathbf{x}_{N_{u}}^{T}\right]^{T}$, where, $\mathbf{x}_{i}$, $i=1, \ldots, N_{u}$, is the signal vector for the $i$-the user. Finally, $\mathbf{w}=\left[\mathbf{w}_{1}^{T}, \ldots, \mathbf{w}_{N_{u}}^{T}\right]^{T} \sim \mathcal{C} \mathcal{N}\left(\mathbf{0}, \sigma_{\mathbf{w}}^{2} \mathbf{I}\right)$ is a $N_{u} N_{r} \times 1$ vector that represents the white Gaussian noise. In more detail, $\mathbf{w}_{i}$, $i=1, \ldots, N_{u}$, is the Gaussian noise observed by the $i$-th user.

The precoding method of interest in the present paper is ZF. The ZF precoder is a suboptimal precoder that offers a good trade-off between complexity and performance [41]. The main characteristic of ZF precoding is the total elimination of interference between different users and between different antennas of the same user. Hence, ZF precoding is an efficient method that can be used for the formation of a MU architecture based on MSR-SM. Therefore, if the channel matrix is expressed as,

$$
\breve{\mathbf{H}}=\boldsymbol{\Xi}^{\frac{1}{2}} \mathbf{H},
$$

where, $\boldsymbol{\Xi}=\operatorname{diag}\left(\xi_{1} \mathbf{I}, \ldots, \xi_{N_{u}} \mathbf{I}\right)$, represents the effect of the system topology, $\mathbf{H}=\left[\mathbf{H}_{1}^{H}, \ldots, \mathbf{H}_{N_{u}}^{H}\right]^{H} \sim \mathcal{C N}(\mathbf{0}, \mathbf{I})$, represents the small scale fading, and $\mathbf{H}_{i}$ represents the small scale fading effect of the $i$-th user, the ZF precoding matrix is written as:

$$
\breve{\mathbf{P}}=\mathbf{H}^{H}\left(\mathbf{H H}^{H}\right)^{-1} \boldsymbol{\Xi}^{-\frac{1}{2}} .
$$

Let the $\mathrm{ZF}$ precoder in (3), the diagonal normalization matrix $\breve{\mathbf{D}}$ can be re-written as, $\breve{\mathbf{D}}=\boldsymbol{\Xi}^{\frac{1}{2}} \mathbf{D}_{\mathrm{MU}}$, where, $\mathbf{D}_{\mathrm{MU}}=$ $\operatorname{diag}\left(d_{1}, \ldots, d_{N_{u} N_{r}}\right)$ is a diagonal matrix. The $i$-th element of the main diagonal of $\mathbf{D}_{\mathrm{MU}}$ is expressed as:

$$
d_{i}=\sqrt{\frac{1}{\left[\left(\mathbf{H H}^{H}\right)^{-1}\right]_{i, i}}}, i=1, \ldots, N_{u} N_{r},
$$

in order to ensure that the instantaneous transmission power is constrained.

In order to gain a better understanding, (2) and (3) can be plugged into (1). By doing so, the received signal at user is expressed as:

$$
\mathbf{y}_{i}=\sqrt{\xi_{i}} \mathbf{D}_{i} \mathbf{x}_{i}+\mathbf{w}_{i}, \quad i=1, \ldots, N_{u},
$$

since $\mathbf{D}_{\mathrm{MU}}=\operatorname{diag}\left(\mathbf{D}_{1} \ldots, \mathbf{D}_{N_{u}}\right)$ is a block diagonal matrix, where $\mathbf{D}_{i}$ is defined as the $i$-th $N_{r} \times N_{r}$ block matrix of the main block diagonal of $\mathbf{D}_{\mathrm{MU}}$. From the structure of $d_{i}$ in (4) and (5), it can be seen that the received signal of the $i$-th user is directly affected by the CSIT of all users.

The inspection of (5) highlights that the choice of the transmitted vector $\mathbf{x}_{i}, i=1, \ldots, N_{u}$, determines the way that information is transmitted to each user. For example, conventional SMX transmission is obtained if all the elements of $\mathbf{x}_{i}$ are drawn from a conventional $M$-ary constellation diagram $\mathcal{M}$. On the other hand, MSR-SM can be obtained by appropriately choosing the symbol vectors $\mathbf{x}_{i}, i=1, \ldots, N_{u}$, as discussed in Section III.

At the users' side, the reconstruction of the transmitted bitstreams is undertaken by detecting the transmitted vectors $\mathbf{x}_{i}$, $i=1, \ldots, N_{u}$. Provided that the $i$-th user is aware of $\xi_{i}$ and $\mathbf{D}_{i}$, this can be implemented at every user independently by using the following ML detector:

$$
\left(\tilde{\mathbf{x}}_{i}\right)=\arg \min _{\mathbf{x}_{i}}\left\|\mathbf{y}_{i}-\sqrt{\xi_{i}} \mathbf{D}_{i} \mathbf{x}_{i}\right\|_{2}^{2}, \quad i=1, \ldots, N_{u} .
$$

\section{Multi-Stream Receive-Spatial Modulation}

The objective of MSR-SM is twofold: i) the simultaneous transmission of $N_{s} \leq N_{r}$ information symbols from the transmitter to the receiver and ii) the transmission of additional information bits via the indices of $N_{s}$ (out of $N_{r}$ ) receive antennas. Assuming ZF precoding, the received signal at each user is given in (5). Hence, by appropriately choosing the structure of the transmit signal vector $\mathbf{x}_{i}$, it is possible to enforce that the noise free received signal $\mathbf{D}_{i} \mathbf{x}_{i}$ has exactly $N_{s}$ non-zero elements and $N_{r}-N_{s}$ zero elements. Let $\mathbf{D}_{i}$ be the diagonal matrix introduced in (5), the non-zero elements of $\mathbf{D}_{i} \mathbf{x}_{i}$ constitute a scaled version of the corresponding non-zero elements of $\mathbf{x}_{i}$. Similarly, the positions of the zero elements of $\mathbf{D}_{i} \mathbf{x}_{i}$ are the same as those of the zero elements of $\mathbf{x}_{i}$. This implies that a portion of binary information can be encoded in the position of the non-zero elements of $\mathbf{x}_{i}$ and consequently in the position of the non-zero elements of the noise free received signal $\mathbf{D}_{i} \mathbf{x}_{i}$.

The general expression of $\mathbf{x}_{i} \in \mathcal{B}_{i}$ for MSR-SM is given in (7), which is available at the top of this page. Here, $\mathcal{B}_{i}$ denotes the set (alphabet) of all possible transmitted symbol vectors to the $i$-th user and $\left\{s_{1}, \ldots, s_{N_{s}}\right\} \in \mathcal{M}$, where $\mathcal{M}$ is the deployed constellation. The positions of the nonzero elements correspond to the indices of the receiving antennas, while the positions of the zero elements correspond to the antennas that do not receive signal. The selection of the combinations of receiving antennas can be optimized in order to minimize the instantaneous Bit Error Rate (BER) or they can be chosen at random. In this paper, for reasons of simplicity and mathematical tractability, the focus is on the latter case.

With these assumptions, the bit-stream to be transmitted in every signaling period is divided in two portions. The first portion, which is of length $k_{1}^{\mathrm{MSR}-\mathrm{SM}}=N_{s} \log _{2}(M)$ bits, is encoded and transmitted using the $N_{s}$ symbols which are drawn from the $M$-ary constellation $\mathcal{M}$. The second portion, which is of length $k_{2}^{\text {MSR-SM }}=\left\lfloor\log _{2}\left(\left(\begin{array}{l}N_{r} \\ N_{s}\end{array}\right)\right)\right\rfloor$ bits, is encoded in the indices of the receive antennas. Here, (.) denotes the binomial coefficient. Therefore, the spectral efficiency of 
MSR-SM is $k_{\text {MSR-SM }}=N_{s} \log _{2}(M)+\left\lfloor\log _{2}\left(\left(\begin{array}{c}N_{r} \\ N_{s}\end{array}\right)\right)\right\rfloor$ bits per channel use (bpcu) per user. In the extreme case, where it holds $N_{s}=N_{r}$, MSR-SM reduces to a spatially multiplexed MIMO architecture with $\mathrm{ZF}$ precoding. In this case, the spectral efficiency is $k_{\mathrm{SMX}}=N_{r} \log _{2}(M)$ bpcu per user.

The inspection of (7) reveals that the sparsity of the transmission alphabet $\mathcal{B}_{i}$ of MSR-SM can be utilized in order to offer lower computational complexity at the transmitter. Provided that the precoding matrix $\breve{\mathbf{P}}$ of (3) is precomputed offline before transmission, the transmitted signal, $\mathbf{s}=\breve{\mathbf{P}} \breve{\mathbf{x}}$, in (1) can be computed with $\mathcal{C}_{t}=N_{t}\left(8 N_{u} N_{s}-2\right)+2 N_{u} N_{s}$ real operations (additions or multiplications). It is clear that as $N_{s}$ takes lower values, the complexity of the transmitter $\mathcal{C}_{t}$ is also reduced. The computational analysis of the detector of MSR-SM is presented in [30]. In that paper, a suboptimal detector with low complexity for MSR-SM is also proposed. The study of the detector of [30] is, however, outside of the scope of the present paper.

\section{Evaluation of the Average Bit Error PROBABILITY}

In this section, the ABEP of each individual user and of the whole MU system are derived. The ABEP of the $i$-th user, $P_{\text {bit }}^{i}(\gamma)$, for a given transmit SNR $\gamma$, can be bounded as follows:

$$
P_{\text {bit }}^{i}(\gamma) \leq \frac{1}{\left|\mathcal{B}_{i}\right| k_{\text {MSR-SM }}} \sum_{\substack{\mathbf{x}_{i} \\ \hat{x}_{i}}} \sum_{\substack{\hat{\mathbf{x}}_{i} \\ \mathbf{x}_{i} \mathbf{x}_{i}}} d\left(\mathbf{x}_{i} \rightarrow \hat{\mathbf{x}}_{i}\right) P_{\mathrm{e}}^{i}\left(\mathbf{x}_{i} \rightarrow \hat{\mathbf{x}}_{i}, \gamma\right),
$$

using the union bound technique [42]. In (8), $P_{\mathrm{e}}^{i}\left(\mathbf{x}_{i} \rightarrow \hat{\mathbf{x}}_{i}, \gamma\right)$ represents the Pairwise Error Probability (PEP) of transmitting $\mathbf{x}_{i}$ to the $i$-th user while the detector decides in favor of the erroneous symbol vector $\hat{\mathbf{x}}_{i}$. The number of different bits between the bit-words represented by $\mathbf{x}_{i}$ and $\hat{\mathbf{x}}_{i}$ is denoted by $d\left(\mathbf{x}_{i} \rightarrow \hat{\mathbf{x}}_{i}\right)$. Furthermore, $\left|\mathcal{B}_{i}\right|=M^{N_{s}} 2\left\lfloor\log _{2}\left(\begin{array}{c}N_{r} \\ N_{s}\end{array}\right)\right\rfloor$ denotes the number of all possible transmitted symbol vectors to the $i$-th user.

The evaluation of (8) requires the knowledge of $P_{\mathrm{e}}^{i}\left(\mathbf{x}_{i} \rightarrow \hat{\mathbf{x}}_{i}, \gamma\right)$, which is the expectation of the instantaneous PEP over all channel realizations. Let the detector of the $i$-th user in (6), a symbol error occurs at this user when, $\mathcal{E}_{i}\left(\mathbf{x}_{i}, \hat{\mathbf{x}}_{i}\right)=\left\{\left\|\mathbf{y}_{i}-\sqrt{\xi_{i}} \mathbf{D}_{i} \mathbf{x}_{i}\right\|_{2}^{2}>\left\|\mathbf{y}_{i}-\sqrt{\xi_{i}} \mathbf{D}_{i} \hat{\mathbf{x}}_{i}\right\|_{2}^{2}\right\}$. In this case, if the statistical distribution of the Gaussian noise of the $i$-th user is taken into account, after some manipulations, the corresponding instantaneous PEP (conditioned on $\mathbf{D}_{i}$ ) is expressed as:

$$
P_{\mathrm{e}}^{i}\left(\mathbf{x}_{i} \rightarrow \hat{\mathbf{x}}_{i}, \gamma \mid \mathbf{D}_{i}^{2}\right)=Q\left(\sqrt{\frac{\mathbf{c}_{i}^{H} \mathbf{D}_{i}^{2} \mathbf{c}_{i}}{2} \xi_{i} \gamma}\right) .
$$

In (9), the vector $\mathbf{c}_{i}$ is defined as $\mathbf{c}_{i}=\mathbf{x}_{i}-\hat{\mathbf{x}}_{i}$. From (9) and the structure of $\mathbf{D}_{i}$, it can be seen that the instantaneous PEP of the $i$-th user depends on the CSIT of all users via $\mathbf{D}_{i}$.

For notational convenience, the following variables are defined:

$$
z_{i}=\mathbf{c}_{i}^{H} \mathbf{D}_{i}^{2} \mathbf{c}_{i}
$$

and

$$
\breve{\gamma}_{i}=\xi_{i} \gamma
$$

In order to evaluate the expectation of (9) over all possible realizations of the diagonal random matrix $\mathbf{D}_{i},(10),(11)$, and the following tight upper bound of the Q-function [43], $Q(x) \leq \frac{1}{6} e^{-2 x^{2}}+\frac{1}{12} e^{-x^{2}}+\frac{1}{4} e^{\frac{-x^{2}}{2}}$, are considered. In this way, the PEP of interest is expressed as:

$$
\begin{aligned}
P_{\mathrm{e}}^{i}\left(\mathbf{x}_{i} \rightarrow \hat{\mathbf{x}}_{i}, \breve{\gamma}_{i}\right) & \leq \frac{1}{6} \mathrm{E}_{z_{i}}\left[e^{-z_{i} \breve{\gamma}_{i}}\right]+\frac{1}{12} \mathrm{E}_{z_{i}}\left[e^{-\frac{z_{i}}{2} \breve{\gamma}_{i}}\right] \\
& +\frac{1}{4} \mathrm{E}_{z_{i}}\left[e^{-\frac{z_{i}}{4} \breve{\gamma}_{i}}\right] .
\end{aligned}
$$

From (12), it can be observed that the Probability Density Function (PDF) of the Random Variable (RV) $z_{i}$ has to be derived. To this end, using an algebraic elaboration on (10), the $\mathrm{RV} z_{i}$ can be re-written as:

$$
z_{i}=\sum_{k=1}^{N_{r}}\left|x_{k}-\hat{x}_{k}\right|^{2} d_{k}^{2}=\sum_{x_{k}-\hat{x}_{k} \neq 0}\left|x_{k}-\hat{x}_{k}\right|^{2} d_{k}^{2},
$$

where, $x_{k}$ and $\hat{x}_{k}, k=1, \ldots, N_{r}$, are the $k$-th elements of $\mathbf{x}_{i}$ and $\hat{\mathbf{x}}_{i}, i=1, \ldots, N_{u}$, respectively. Furthermore, $d_{k}$, $k=1, \ldots, N_{r}$, is the $k$-th element of the main diagonal of $\mathbf{D}_{i}$.

Usually, in the literature, the RVs $d_{k}^{2}$ are assumed to be statistically independent in order to simplify the mathematical analysis $[44,45]$. This assumption is, however, in contradiction with the structure of $d_{k}^{2}=1 /\left[\left(\mathbf{H}_{i} \mathbf{H}_{i}^{H}\right)^{-1}\right]_{k, k}$. In fact, the realization of every $\mathrm{RV} d_{k}^{2}$ occurs using the same mathematical operations on the same random matrix $\mathbf{H}_{i}$. More specifically, the following holds [46]:

$$
d_{k}^{2}=\frac{1}{\left[\operatorname{adj}\left(\mathbf{H}_{i} \mathbf{H}_{i}^{H}\right)\right]_{k, k}} \operatorname{det}\left(\mathbf{H}_{i} \mathbf{H}_{i}^{H}\right),
$$

where, $\operatorname{adj}(\cdot)$ is the adjoint matrix and $\operatorname{det}(\cdot)$ is the matrix determinant. The inspection of (14) shows that for different values of $k=1, \ldots, N_{r}$, the realization of the RVs $d_{k}^{2}$ affects one another, since they are produced via the same mathematical formula using the same random elements of $\mathbf{H}_{i}$. This implies that the RVs $d_{k}^{2}$ are dependent. An empirical confirmation for the previous argument can be obtained by computing the Pearson product-moment correlation coefficient between any pair of the previous RVs using multiple samples. In this way, it can be shown that the Pearson product-moment correlation coefficient takes non-zero values. The analytical evaluation of these correlation coefficients is difficult to be obtained, since it requires the joint PDF between each pair of the RVs $d_{k}^{2}$. An additional confirmation is provided in Section VI-A, where the empirical PDF of $z_{i}$ is depicted against the theoretical PDF derived below. Hence, it can be concluded that the RVs $d_{k}^{2}$ are statistically dependent.

Due to the dependence between the RVs $d_{k}^{2}$, a different approach, compared to the state-of-the-art literature, is proposed: we take into account that $d_{k}^{2}, k=1, \ldots, N_{r}$, are dependent and correlated gamma RVs.

For notational convenience, the variables

$$
b_{j}=\left|c_{k}\right|^{2}=\left|x_{k}-\hat{x}_{k}\right|^{2},
$$

and

$$
X_{j}=d_{k}^{2},
$$




$$
f_{z_{i}}(x)=\left[\prod_{l=1}^{N_{i}}\left(\frac{\breve{\alpha}_{1}}{\breve{\alpha}_{l}}\right)^{L_{\mathrm{MU}}}\right]\left[\sum_{k=0}^{+\infty} \frac{\breve{\delta}_{k} x^{N_{i} L_{\mathrm{MU}}+k-1} e^{-\frac{x}{\alpha_{1}}}}{\breve{\alpha}_{1}^{N_{i} L_{\mathrm{MU}}+k} \Gamma\left(N_{i} L_{\mathrm{MU}}+k\right)}\right] H_{0}(x)
$$

$$
\breve{\delta}_{k+1}= \begin{cases}1, & k=-1, \\ \frac{k}{k+1} \sum_{i=1}^{k+1}\left[\sum_{j=1}^{N}\left(1-\frac{\breve{\alpha}_{1}}{\breve{\alpha}_{j}}\right)^{i}\right] \breve{\delta}_{k+1-i}, & k=0,1,2, \ldots\end{cases}
$$

$j=1, \ldots, N_{i}$, are introduced only for those values of $k$ in (13) for which it holds that $c_{k}=x_{k}-\hat{x}_{k} \neq 0$. Here, $c_{k}$ is the $k$-th element of $\mathbf{c}_{i}$. In addition, $N_{i}$ is the number of non zero elements of $\mathbf{c}_{i}$. Thus, the value of $N_{i}$ depends on the considered pair of $\mathbf{x}_{i}$ and $\hat{\mathbf{x}}_{i}$. Therefore, (13) can be re-written as:

$$
z_{i}=\sum_{j=1}^{N_{i}} b_{j} X_{j}=\sum_{j=1}^{N_{i}} Z_{j}
$$

In (17), $Z_{j}$ is defined as:

$$
Z_{j}=b_{j} X_{j}
$$

The PDF of $d_{k}^{2}$ is explicitly derived in [46] as a gamma distribution with $d_{k}^{2} \sim \operatorname{Gamma}\left(L_{\mathrm{MU}}, 1\right)$ and

$$
L_{\mathrm{MU}}=N_{t}-N_{u} N_{r}+1 .
$$

Consequently, given that $X_{j}=d_{k}^{2}, X_{j}$ follows the same distribution. Therefore, the RVs $Z_{j}=b_{j} X_{j}, j=1, \ldots, N_{j}$, are distributed as $Z_{j} \sim \operatorname{Gamma}\left(L_{\mathrm{MU}}, b_{j}\right)$, with a PDF given by:

$$
f_{Z_{j}}(x)=\frac{1}{b_{j}^{L_{\mathrm{MU}}} \Gamma\left(L_{\mathrm{MU}}\right)} x^{L_{\mathrm{MU}}-1} e^{\frac{x}{b_{j}}} H_{0}(x),
$$

Here, $H_{0}(x)$ is the Heaviside step function defined as, $H_{0}(x)=0$ for $x<0$ and $H_{0}(x)=1$ for $x \geq 0$.

Since $X_{j}, j=1, \ldots, N_{j}$, are correlated RVs, also, $Z_{j}=$ $b_{j} X_{j}, j=1, \ldots, N_{j}$ are correlated RVs. This implies that $z_{i}$ is a RV which is equal to the sum of correlated Gamma RVs. For this reason and based on [47, Corollary 1], the PDF of $z_{i}$ is given in (21) at the top of this page. Note that, because of the correlation between the different pairs of RVs $Z_{j}$, the derivations provided below are significantly different than the corresponding derivations of a conventional MIMO system which deploys ZF detection. Such an example is the performance analysis presented in [38]. In fact, in a conventional MIMO system with ZF detection, the detection process of the parallel symbol streams decouples. Hence, each parallel symbol stream can be detected independently. Therefore, the correlation between the RVs which represent the instantaneous receive SNR of each parallel symbol stream does not need to be considered.

In (21), $\breve{\alpha}_{l}, l=1, \ldots, N_{i}$, are the eigenvalues of:

$$
\breve{\mathbf{A}}=\breve{\mathbf{B}} \breve{\mathbf{R}}
$$

in ascending order, and $\breve{\mathbf{B}}$ is the diagonal matrix

$$
\breve{\mathbf{B}}=\operatorname{diag}\left(b_{1}, \ldots, b_{N_{i}}\right),
$$

where $b_{l}, l=1, \ldots, N_{i}$, is the square of the absolute value of the $l$-th non zero element of $\mathbf{c}_{i}$. Moreover, $\breve{\mathbf{R}}$ is a $N_{i} \times N_{i}$ matrix defined as:

$$
\breve{\mathbf{R}}=\left[\begin{array}{cccc}
1 & \sqrt{\rho_{c}} & \cdots & \sqrt{\rho_{c}} \\
\sqrt{\rho_{c}} & \ddots & \ddots & \vdots \\
\vdots & \ddots & \ddots & \sqrt{\rho_{c}} \\
\sqrt{\rho_{c}} & \cdots & \sqrt{\rho_{c}} & 1
\end{array}\right]
$$

where, $\rho_{c}$ is the Pearson product-moment correlation coefficient between any pair of two different RVs of the main diagonal of $\mathbf{D}_{i}^{2}$. The inspection of the structure of $d_{k}^{2}$ shows that the Pearson product-moment correlation coefficient between every pair of two different RVs of the main diagonal of $\mathbf{D}_{i}^{2}$ takes the value of $\rho_{c}$. Finally, $\breve{\delta}_{k}, k=0,1,2, \ldots$, are given in (25) at the top of this page.

Let the PDF of $z_{i}$ given in (21), the evaluation of (12) over all possible realizations of $z_{i}$ can be performed by evaluating expectations of the following form, $g(y)=\mathrm{E}_{z_{i}}\left[e^{-y \breve{\gamma}_{i} z_{i}}\right]$. Here, $y$ is a deterministic scalar. Thus, the evaluation of the previous expectation is given as:

$$
\begin{aligned}
g(y) & =\mathrm{E}_{z_{i}}\left[e^{-y \breve{\gamma}_{i} z_{i}}\right]=\int_{-\infty}^{+\infty} e^{-y \breve{\gamma}_{i} x} f_{z_{i}}(x) d x \\
& =\left[\prod_{l=1}^{N_{i}}\left(\frac{\breve{\alpha}_{1}}{\breve{\alpha}_{l}}\right)^{L_{\mathrm{MU}}}\right]\left[\int_{0}^{+\infty} e^{-y \breve{\gamma}_{i} x}\right. \\
& \left.\times \sum_{k=0}^{+\infty} \frac{\breve{\delta}_{k} x^{N_{i} L_{\mathrm{MU}}+k-1} e^{-\frac{x}{\check{\alpha}_{1}}}}{\breve{\alpha}_{1}^{N_{i} L_{\mathrm{MU}}+k} \Gamma\left(N_{i} L_{\mathrm{MU}}+k\right)} d x\right] \\
& =\left[\prod_{l=1}^{N_{i}}\left(\frac{\breve{\alpha}_{1}}{\breve{\alpha}_{l}}\right)^{L_{\mathrm{MU}}}\right] \sum_{k=0}^{+\infty}\left[\frac{\breve{\delta}_{k}}{\breve{\alpha}_{1}^{N_{i} L_{\mathrm{MU}}+k} \Gamma\left(N_{i} L_{\mathrm{MU}}+k\right)}\right. \\
& \left.\times \int_{0}^{+\infty} x^{N_{i} L_{\mathrm{MU}} 1+k-1} e^{-\left(y \breve{\gamma}_{i}+\frac{1}{\check{\alpha}_{1}}\right) x} d x\right]
\end{aligned}
$$

From the integration formula in [48, p.346, 3.381, 4], we have:

$$
\int_{0}^{+\infty} x^{\nu-1} e^{-\mu x} d x=\mu^{-\nu} \Gamma(\nu),
$$

where, $\nu>0$ and $\operatorname{Re}\{\mu\}>0$, and $\Gamma(\cdot)$ denotes the incomplete gamma function defined in [48, p. 899]. By plugging (27) in (26) and with the aid of some manipulations, we have:

$$
\begin{aligned}
g(y) & =\left[\prod_{l=1}^{N_{i}}\left(\frac{\breve{\alpha}_{1}}{\breve{\alpha}_{l}}\right)^{L_{\mathrm{MU}}}\right]\left(y \breve{\alpha}_{1} \breve{\gamma}_{i}+1\right)^{-N_{i} L_{\mathrm{MU}}} \\
& \times \sum_{k=0}^{+\infty} \breve{\delta}_{k}\left(y \breve{\alpha}_{1} \breve{\gamma}_{i}+1\right)^{-k} .
\end{aligned}
$$




$$
\begin{aligned}
P_{\mathrm{e}}^{i}(\mathbf{x} \rightarrow \hat{\mathbf{x}}, \gamma) \leq & \frac{\left[\prod_{l=1}^{N_{i}}\left(\frac{\breve{\alpha}_{1}}{\breve{\alpha}_{l}}\right)^{L_{\mathrm{MU}}}\right]}{6}\left(\breve{\alpha}_{1} \xi_{i} \gamma+1\right)^{-N_{i} L_{\mathrm{MU}}} \sum_{k=0}^{+\infty} \breve{\delta}_{k}\left(\breve{\alpha}_{1} \xi_{i} \gamma+1\right)^{-k} \\
& +\frac{\left[\prod_{l=1}^{N_{i}}\left(\frac{\breve{\alpha}_{1}}{\breve{\alpha}_{l}}\right)^{L_{\mathrm{MU}}}\right]}{12}\left(\frac{\breve{\alpha}_{1}}{2} \xi_{i} \gamma+1\right)^{-N_{i} L_{\mathrm{MU}}} \sum_{k=0}^{+\infty} \breve{\delta}_{k}\left(\frac{\breve{\alpha}_{1}}{2} \xi_{i} \gamma+1\right)^{-k} \\
& +\frac{\left[\prod_{l=1}^{N_{i}}\left(\frac{\breve{\alpha}_{1}}{\breve{\alpha}_{l}}\right)^{L_{\mathrm{MU}}}\right]}{4}\left(\frac{\breve{\alpha}_{1}}{4} \xi_{i} \gamma+1\right)^{-N_{i} L_{\mathrm{MU}}} \sum_{k=0}^{+\infty} \breve{\delta}_{k}\left(\frac{\breve{\alpha}_{1}}{4} \xi_{i} \gamma+1\right)^{-k} .
\end{aligned}
$$

$$
\begin{aligned}
P_{i}^{+\infty}\left(\mathbf{x}_{i} \rightarrow \hat{\mathbf{x}}_{i}, \gamma\right) & \lesssim \frac{\left[\prod_{l=1}^{N_{i}}\left(\frac{\breve{\alpha}_{1}}{\breve{\alpha}_{l}}\right)^{L_{\mathrm{MU}}}\right]}{2}\left(\frac{\breve{\alpha}_{1}}{4} \xi_{i} \gamma+1\right)^{-N_{i} L_{\mathrm{MU}}} \\
& =\left[\sqrt[N_{i} L_{\mathrm{MU}}]{\frac{2}{\prod_{l=1}^{N_{i}}\left(\frac{\breve{\alpha}_{1}}{\check{\alpha}_{l}}\right)^{L_{\mathrm{MU}}}}} \frac{\breve{\alpha}_{1}}{4} \xi_{i} \gamma+\sqrt{\frac{2}{L_{i} L_{\mathrm{MU}}}}\right]^{-N_{i} L_{\mathrm{MU}}} \\
& \approx \gamma^{-N_{i} L_{\mathrm{MU}}}\left[\frac{\prod_{l=1}^{N_{i}}\left(\frac{\breve{\alpha}_{1}}{\prod_{l}}\right)^{N_{\mathrm{MU}}}\left(\frac{\breve{\alpha}_{1}}{\check{\alpha}_{l}}\right)^{L_{\mathrm{MU}}}}{2}\left(\frac{\breve{\alpha}_{1}}{4} \xi_{i}\right)^{-N_{i} L_{\mathrm{MU}}}\right]+\mathrm{o}\left(\gamma^{-N_{i} L_{\mathrm{MU}}}\right) \\
& \leq\left[\frac{\breve{\alpha}_{1} N_{i} L_{\mathrm{MU}}}{4} \xi_{i} \gamma\right]^{-N_{i} L_{\mathrm{MU}}}+\mathrm{o}\left(\gamma^{-N_{i} L_{\mathrm{MU}}}\right) .
\end{aligned}
$$

Since $\breve{\gamma}_{i}=\xi_{i} \gamma$ and using the result from (28), the PEP of the $i$-th user is given in (29) at the top of this page. Thus, the evaluation of the ABEP of the $i$-th user follows from (8), by using (29).

In addition to the performance of each user, the whole system performance is of interest. A metric that is able to evaluate the whole system performance is the system ABEP. Assuming that the detection process at each user is performed independently, the system ABEP is expressed as:

$$
P_{\text {bit }}^{\text {System }}(\gamma)=\frac{1}{N_{u}} \sum_{i}^{N u} P_{\text {bit }}^{i}\left(\gamma_{i}\right) .
$$

An upper bound of (30) can be obtained by using the upper bound of the PEP, $P_{\mathrm{bit}}^{i}\left(\gamma_{i}\right)$, of each user given in (8).

\section{ANALysis of Diversity ORder ANd CODING Gain}

In the high SNR regime, the user and system performance can be characterized in terms of diversity order and coding gain. In [49], the diversity order and coding gain are obtained from the metric of Symbol Error Rate (SER). Therefore, in order to be perfectly aligned with [49], the focus in this section is on the metric of SER. We start by analyzing these performance measures for the $i$-th user, $i=1, \ldots, N_{u}$ and then generalize the analysis from the system standpoint based on (30).

In order to compute the diversity order and coding gain of the $i$-th user, a high SNR approximation for the PEPs of the $i$-th user is needed. By using mathematical steps similar to Section IV and based on the Chernoff bound of the $Q$-function, $Q(x) \leq \frac{1}{2} e^{-\frac{x^{2}}{2}}$, the PEP of the $i$-th user can be bounded as:

$$
\begin{aligned}
P_{\mathrm{e}}^{i}\left(\mathbf{x}_{i} \rightarrow \hat{\mathbf{x}}_{i}, \gamma\right) & \leq \frac{\left[\prod_{l=1}^{N_{i}}\left(\frac{\breve{\alpha}_{1}}{\alpha_{l}}\right)^{L_{\mathrm{MU}}}\right]}{2}\left(\frac{\breve{\alpha}_{1}}{4} \xi_{i} \gamma+1\right)^{-N_{i} L_{\mathrm{MU}}} \\
& \times \sum_{k=0}^{+\infty} \breve{\delta}_{k}\left(\frac{\breve{\alpha}_{1}}{4} \xi_{i} \gamma+1\right)^{-k}
\end{aligned}
$$

From (31), a high SNR $(\gamma \rightarrow+\infty)$ approximation of the PEP of the $i$-th user can be obtained as given below. If the SNR approaches to infinity, only the smallest value of the exponent $k$ in (31) needs to be considered, which is equal to one. In this way, using the previous simplification, (31) can be further approximated as shown in (32) at the top of this page. Note that the last step in (32) follows from the inequality:

$$
\prod_{l=1}^{N_{i}}\left(\frac{\breve{\alpha}_{1}}{\breve{\alpha}_{l}}\right)^{L_{\mathrm{MU}}} \leq 1
$$

which holds because $\breve{\alpha}_{l}, l=1, \ldots, N_{i}$, are the eigenvalues of $\breve{A}$ in (22) in ascending order.

In this case, a high SNR approximation of the SER of the $i$-th user is obtained as follows:

$$
\mathrm{SER}_{i}^{+\infty} \lesssim \frac{1}{|\mathcal{B}|} \sum_{\mathbf{x}_{i}} \sum_{\substack{\hat{\mathbf{x}}_{i} \\ \mathbf{x}_{i} \neq \mathbf{x}_{i}}} P_{i}^{+\infty}\left(\mathbf{x}_{i} \rightarrow \hat{\mathbf{x}}_{i}, \gamma\right) .
$$


In (34), $\mathcal{B}$ denotes the set of all possible transmitted symbol vectors to a generic user.

It can be observed that the high SNR approximation of the SER in (34) is a linear combination of $P_{i}^{+\infty}\left(\mathbf{x}_{i} \rightarrow \hat{\mathbf{x}}_{i}, \gamma\right)$, as given in (32), for all possible pairs of $\mathbf{x}_{i}$ and $\hat{\mathbf{x}}_{i}$. Therefore, as $\gamma \rightarrow+\infty$, the slope of (34) is determined by the smallest exponent of $\gamma$ in (32), i.e. $N_{i} L_{\mathrm{MU}}$. The smallest value of $N_{i} L_{\mathrm{MU}}$ occurs when $N_{i}=1$. In fact, the dominant addends of (34) are those for which $N_{i}=1$. Therefore, the high SNR approximation of the SER in (34) can be further approximated by considering only these dominant addends. In addition, the careful inspection of (32) shows that the matrix $\breve{\mathbf{A}}$ in (22) reduces to a scalar if $N_{i}=1$. This implies $\breve{\alpha}_{1}=b_{1}$, where $b_{1}$ is given in (15).

With this simplification at hand, a more insightful approximation of (34) can be obtained. More specifically, from (32), (34) can be expressed as:

$$
\begin{aligned}
\mathrm{SER}_{i}^{+\infty} & \lesssim \frac{1}{|\mathcal{B}|} \sum_{\mathbf{x}_{i}} \sum_{n=1}^{N_{s}} \sum_{x \in \mathcal{M}} \sum_{\substack{\hat{x} \in \mathcal{M} \\
\hat{x} \neq x}}\left[\frac{|x-\hat{x}|^{2} \sqrt[L_{\mathrm{MU}}]{2}}{4} \xi_{i} \gamma\right]^{-L_{\mathrm{MU}}} \\
& +\sum_{k=2}^{N_{s}} \mathrm{o}\left(\gamma^{-k L_{\mathrm{MU}}}\right) .
\end{aligned}
$$

By using a line of thought similar to [42, Chapter 5.2.9], an upper bound for (35) is obtained by retaining only the minimum distance, denoted by $d_{\min }$, between every pair of the constellation points $\{x, \hat{x}\} \in \mathcal{M}$. By doing so, after some algebraic manipulations of the summations in (35), the following high SNR approximation of the SER of the $i$-th user is obtained:

$$
\begin{aligned}
\mathrm{SER}_{i}^{+\infty} & \lesssim\left[\frac{d_{\mathrm{min}}^{2}}{4} \sqrt[L_{\mathrm{MU}}]{\frac{2}{N_{s}\left(M^{2}-M\right)}} \xi_{i} \gamma\right]^{-L_{\mathrm{MU}}} \\
& +\sum_{k=2}^{N_{s}} \mathrm{o}\left(\gamma^{-k L_{\mathrm{MU}}}\right)
\end{aligned}
$$

The bound in (36) may be loose for high values of the constellation order $M$ [42, Chapter 5.2.9]. However, it is conveniently formulated for providing insightful information on the achievable diversity order and coding gain. If $M$ is large, if needed, a tighter bound may be obtained by following the guidelines in [42, Chapter 5.2.9].

Based on the definitions of the diversity order and coding gain available in [49], the inspection of (36) reveals that the diversity order of the $i$-th user is:

$$
d_{i}=L_{\mathrm{MU}}
$$

and that the corresponding coding gain is:

$$
c_{i}=\frac{d_{\min }^{2}}{4} \sqrt[L_{\mathrm{MU}}]{\frac{2}{N_{s}\left(M^{2}-M\right)}} \xi_{i} .
$$

Since $L_{\mathrm{MU}}=N_{t}-N_{u} N_{r}+1$, from (37) it follows that the diversity order of the $i$-user does not depend on large-scale channel effect, but only on the system size (the number of transmit antennas $N_{r}$, the number of users $N_{u}$, and the number of receive antennas per user $N_{r}$ ). In contrast, (38) shows that the coding gain of the $i$-th user depends on the system size, the number of parallel data streams $N_{s}$, the constellation size $\mathcal{M}$ (via $d_{\min }$ and $M$ ), and $\xi_{i}$ which represents the large-scale channel effect. Here, it is indirectly assumed that the largescale channel effect is deterministic.

From the system-level standpoint, the diversity order can be computed by approximating the system SER for high SNR $(\gamma \rightarrow+\infty)$ as follows:

$$
\begin{aligned}
\operatorname{SER}_{\text {System }}^{+\infty} & =\frac{1}{N_{u}} \sum_{i=1}^{N_{u}} \mathrm{SER}_{i}^{+\infty} \\
& \lesssim \frac{1}{N_{u}} \sum_{i=1}^{N_{u}}\left[\frac{d_{\mathrm{min}}^{2}}{4} \sqrt[L_{\mathrm{MU}}]{\frac{2}{N_{s}\left(M^{2}-M\right)}} \xi_{i} \gamma\right]^{-L_{\mathrm{MU}}} \\
& +\sum_{k=2}^{N_{s}} \mathrm{o}\left(\gamma^{-k L_{\mathrm{MU}}}\right)
\end{aligned}
$$

where, the last step in (39) exploits the high SNR approximation in (36). In order to express (39) in a convenient form that explicitly provides information on the diversity order and coding gain, an upper bound based on the smallest value of $\xi_{i}$, $i=1, \ldots, N_{u}$ is used. More specifically, the following holds:

$$
\begin{aligned}
\operatorname{SER}_{\text {System }}^{+\infty} & \lesssim\left[\frac{d_{\min }^{2}}{4} \sqrt[L_{\mathrm{MU}}]{\frac{2}{N_{s}\left(M^{2}-M\right)}} \xi_{\min } \gamma\right]^{-L_{\mathrm{MU}}} \\
& +\sum_{k=2}^{N_{s}} \mathrm{o}\left(\gamma^{-k L_{\mathrm{MU}}}\right)
\end{aligned}
$$

where, $\xi_{\min }=\min \left(\xi_{1}, \ldots, \xi_{N_{u}}\right)$. From (40) and [49], it follows that the system diversity order is:

$$
d_{\text {System }}=L_{\mathrm{MU}},
$$

and the corresponding coding gain is:

$$
c_{\text {System }}=\frac{d_{\min }^{2}}{4} \sqrt[L_{\mathrm{MU}}]{\frac{2}{N_{s}\left(M^{2}-M\right)}} \xi_{\text {min }} .
$$

Comparing (41) and (42) with (37) and (38), respectively, we conclude that the diversity order and the coding gain from the user and system standpoints are the same. The main difference is that the system-level coding gain in (42) is dominated by the large-scale channel effect of the user having the weakest channel, i.e., the smallest value of $\xi_{i}$, $i=1, \ldots, N_{u}$.

Based on the obtained expressions of the diversity order and coding gain, the proposed transmission scheme can be compared against the conventional MIMO broadcast channel. To this end, it is worth noting that the proposed mathematical framework is directly applicable to the conventional MIMO broadcast channel by simply setting $N_{s}=N_{r}$. Therefore, (37), (38), (41), and (42) can be directly used for comparing MU MSR-SM and the conventional MIMO broadcast channel.

From (37) and (41), in particular, we conclude that the diversity order is independent of $N_{s}$. As a result, both schemes achieve the same diversity order. As for the conventional MIMO broadcast channel, this conclusion is in agreement with the results available in [50]. This further validates the 
correctness of our mathematical framework.

The comparison of (38) and (42), on the other hand, brings to our attention that the coding gain depends on $N_{s}$. Therefore, the coding gain of MU MSR-SM, where, in general, $N_{s}<N_{r}$ holds, is different from the coding gain of the conventional MIMO broadcast channel, where $N_{s}=N_{r}$. Since both schemes offer the same diversity order, the scheme providing the highest coding gain also results in the lowest BER. Hence, the superiority of a scheme compared to the other can be assessed by a direct inspection of the following coding gain ratio:

$$
\begin{aligned}
\lambda_{i} & =\frac{c_{i}^{\mathrm{MSR}-\mathrm{SM}}}{c_{i}^{\mathrm{SMX}}} \\
& =\left(\frac{d_{\mathrm{min}}^{\mathrm{MSR}-\mathrm{SM}}}{d_{\mathrm{min}}^{\mathrm{SMX}}}\right)^{2} \sqrt[L_{\mathrm{MU}}]{\frac{N_{r}\left(M_{\mathrm{SMX}}^{2}-M_{\mathrm{SMX}}\right)}{N_{s}\left(M_{\mathrm{MSR}-\mathrm{SM}}^{2}-M_{\mathrm{MSR}-\mathrm{SM}}\right)}} .
\end{aligned}
$$

By appropriately choosing the constellation orders $M_{\mathrm{MSR}-\mathrm{SM}}$ and $M_{\mathrm{SMX}}$ for MU MSR-SM and for the conventional MIMO broadcast channel, respectively, the same spectral efficiency can be guaranteed. In (43), the coding gain of the $i$-th user of MU MSR-SM is denoted by $c_{i}^{\mathrm{MSR}-\mathrm{SM}}$ and the coding gain of the same user in the conventional MIMO broadcast channel is denoted by $c_{i}^{\mathrm{SMX}}$. Furthermore, $d_{\mathrm{min}}^{\mathrm{MSR}-\mathrm{SM}}$ and $d_{\mathrm{min}}^{\mathrm{SMX}}$ denote the minimum distance between every pair of points of the adopted signal constellations for MU MSR-SM and for the conventional MIMO broadcast channel, respectively. From (43), it follows that MU MSR-SM performs better than the conventional MIMO broadcast channel if $\lambda_{i}>1$.

If $M_{\mathrm{MSR}-\mathrm{SM}}=M_{\mathrm{SMX}}$, a direct inspection of (43) reveals that $\lambda_{i}>1$ and that it increases as $N_{s}$ decreases. This is also supported by the fact that as $N_{s}$ is reduced, $d_{\min }^{\mathrm{MSR}-\mathrm{SM}}$ is increased. This happens because, for a fair comparison, the power of $\mathbf{x}$ and $\mathbf{x}_{i}$ should be irrespective of $N_{s}$. Therefore, the distances between the points of a deployed constellation are increased as $N_{s}$ is reduced. As a result, in this case, MU MSR-SM outperforms the conventional MIMO broadcast channel.

In general, however, it holds that $M_{\mathrm{MSR}-\mathrm{SM}} \neq M_{\mathrm{SMX}}$. In this case, usually, MU MSR-SM deploys a constellation of higher order in order to achieve the same spectral efficiency as the conventional MIMO broadcast channel. Therefore, the minimum distance, $d_{\mathrm{min}}^{\mathrm{MSR}-\mathrm{SM}}$, between every pair of points of the deployed constellation is decreased. Hence, although the decrease of $N_{s}$ have a positive effect on the increase of the value of $\lambda_{i}$, the combined effect of increasing the constellation order and consequently reducing $d_{\min }^{\mathrm{MSR}-\mathrm{SM}}$ may result in lower values of $\lambda_{i}$.

In this case, a direct analysis of (43) is more difficult. The ratio $\lambda_{i}$ can, however, be numerically computed. Table I provides typical values of $\lambda_{i}$ in $\mathrm{dB}$ scale, by assuming the same spectral efficiency for both schemes. The inspection of Table I shows that, for a group of system setups $\left(N_{t}=20\right.$, $N_{r}=4, N_{u}=4, N_{s}=3 ; N_{t}=20, N_{r}=5, N_{u}=4$, $N_{s}=4$; and $N_{t}=16, N_{r}=4, N_{u}=4, N_{s}=3$ ), MU MSR-SM provides a higher coding gain than the conventional
TABLE I

CODING GAIN OF MU MSR-SM, BASED ON (43), WITH RESPECT TO THE CONVENTIONAL MIMO BROADCAST CHANNEL $\left(N_{s}=N_{r}\right)$.

\begin{tabular}{c|c|c|c}
\hline \hline System Configuration & $N_{s}$ & $k_{\text {user }}$ (bpsp) & $\lambda_{i}$ (in dB) \\
\hline \hline$N_{t}=20, N_{r}=4, N_{u}=4$ & 3 & 8 & 1.49 \\
$N_{t}=20, N_{r}=4, N_{u}=4$ & 2 & 8 & -2.49 \\
$N_{t}=20, N_{r}=4, N_{u}=4$ & 1 & 8 & -11.05 \\
$N_{t}=20, N_{r}=5, N_{u}=4$ & 4 & 10 & 1.93 \\
$N_{t}=16, N_{r}=4, N_{u}=4$ & 3 & 8 & 2.49 \\
$N_{t}=10, N_{r}=2, N_{u}=4$ & 1 & 8 & -2.98 \\
\hline \hline
\end{tabular}

MIMO broadcast channel. More specifically, the coding gain is in the range between 1 and $2.49 \mathrm{~dB}$. On the other hand, for the rest of the system setups, the conventional MIMO broadcast channel provides a higher coding gain. As a result, (43) can be used for the system optimization and for ensuring that MSR-SM is superior to the state-of-the-art. Note that, as shown in Section II, smaller values of $N_{s}$ result in lower computational complexity at the transmitter. Therefore, in terms of coding gain and complexity at the transmitter, the optimal way for selecting the value of $N_{s}$ is to find the smallest one for which it holds that $\lambda_{i} \geq 1$.

\section{Simulation Results AND Discussion}

The objective of this section is twofold. First, to validate the theoretical results of Sections IV and V using simulation results. Second, to provide a performance comparison between MU MSR-SM and the benchmark MIMO broadcast channel. In this latter case, in particular, $N_{s}=N_{r}$ is assumed and no SM is used. More specifically, the benchmark system conveys information to the $N_{u}$ remote users by establishing $N_{r}$ parallel and non-interfering data streams to each one of them. In all studied scenarios, the number of users is equal to $N_{u}=4$. As described in Section II, the wireless channel of the $i$-th user, $i=1, \ldots, N_{u}$, is generated following a complex Gaussian distribution $\left(\breve{\mathbf{H}}_{i} \sim \mathcal{C N}\left(\mathbf{0}, \xi_{i} \mathbf{I}\right)\right)$. In more detail, $\xi_{i}, i=1, \ldots, N_{u}$, is set equal to $1,0.75,0.5$, and 0.25 , for user $1,2,3$, and 4 , respectively. This choice allows us to demonstrate how the large-scale channel effect (system topology) affects the performance of different users. Values of $\xi_{i}$ which are close to one model strong channels, while as $\xi_{i}$ is reduced and approaches zero, less strong channels are modeled. Note that, as it is a common assumption in the literature $[41,45,46,51-54]$, the effect of shadowing is not considered. Thus, the value of $\xi_{i}$ is solely determined by the transmission distance (pathloss). More specifically, for the $i$ th user and at a normalized distance $r_{i}=\bar{r}_{i} / r_{0}$, where $\bar{r}_{i}$ is the transmission distance and $r_{0}$ is a given reference distance, the value of $\xi_{i}$ is given as, $\xi_{i}=1 / r_{i}^{\alpha}$. Here, $\alpha \geq 2$ is the pathloss exponent. Therefore, for $\alpha=2$, the previous values of $\xi_{i}$ correspond to the following normalized distances of 1 , $1.154,1.142$, and 2, respectively. For a fair comparison, the $M$-ary constellations of both schemes are normalized such that $\mathrm{E}_{\mathbf{x}}[\mathbf{x}]=1$ and $\mathrm{E}_{\mathbf{x}_{i}}\left[\mathbf{x}_{i}\right]=\frac{1}{N_{u}}$. So, the transmit SNR of the whole system is $\gamma=\frac{1}{\sigma_{\mathbf{w}}^{2}}$. 


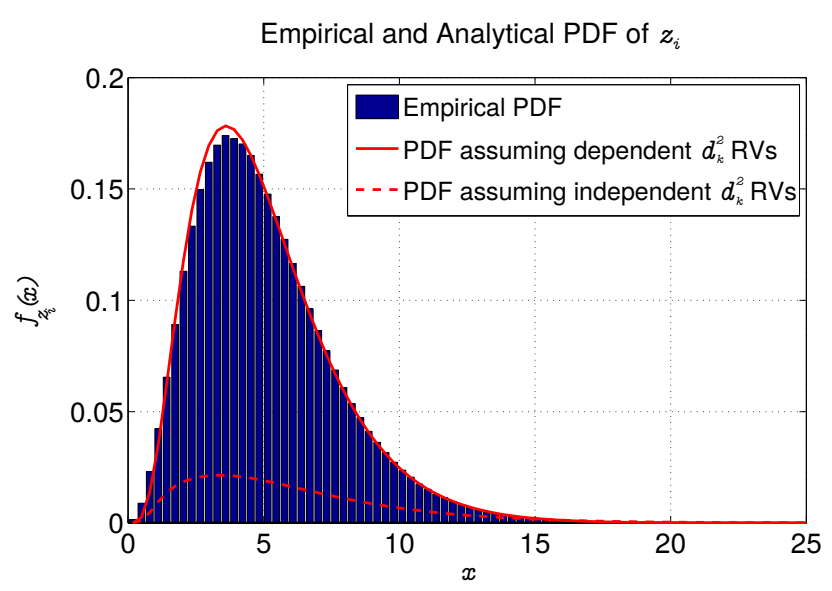

Fig. 1. Illustration of empirical and analytical PDF of (17) by assuming that: i) the RVs $d_{k}, k=1, \ldots, 2$ are statistically dependent and ii) they are independent. Setup: $\mathbf{H} \sim \mathcal{C N}\left(\mathbf{0}_{2 \times 4}, \mathbf{I}_{2 \times 4}\right)$; and ii) $b_{1}=0.5$ and $b_{1}=1.2$.

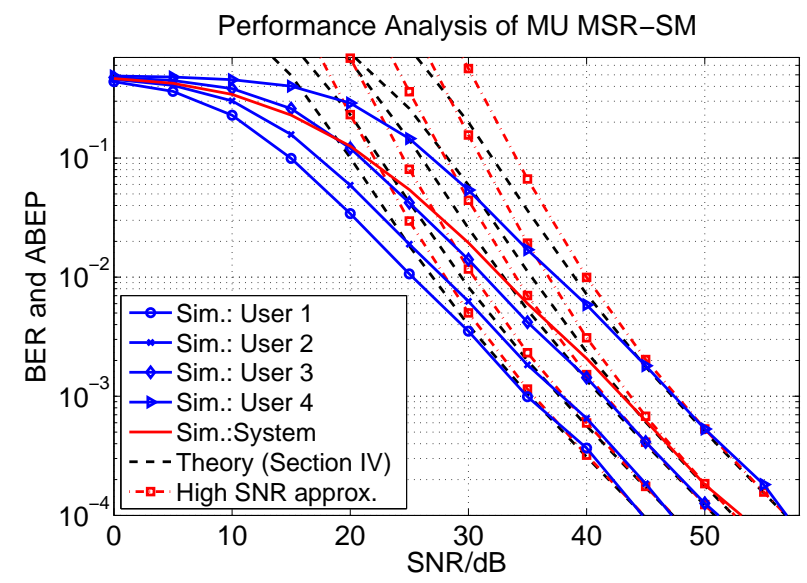

Fig. 2. Performance analysis of MU R-SM $\left(N_{s}=1\right)$ for four users, when ZF with P-CSIT is employed: simulation results vs. the bounds in Section IV. The high SNR approximation of the ABEP is calculated using the PEP given in (32). Setup: $N_{t}=16, N_{r}=4, \xi_{i}, i=1, \ldots, N_{u}$, takes the values 1 , $0.75,0.5$, and 0.25 for the user $1,2,3$, and 4 , respectively.

\section{A. Validation of (21)}

Section IV provides the ABEP of MU MSR-SM by using the PDF of $z_{i}$ given in (21). The derivation of (21) is based on the fact that the RVs $d_{k}^{2}, k=1, \ldots, N_{r}$, are statistically correlated. In order to confirm this, Fig. 1 illustrates the empirical PDF of (17) and compares it against its analytical expression in (21). In addition, Fig. 1 shows the analytical PDF of (17) under the incorrect assumption that $d_{k}^{2}, k=1, \ldots, N_{r}$, are statistically independent RVs, as usually considered in the literature for mathematical tractability. If this assumption was valid, the PDF of (17) could be directly obtained by using the result from [47, Theorem 1]. From Fig. 1, we observe that the theoretical PDF of (17) perfectly matches its empirical PDF. In contrast, when the RVs $d_{k}^{2}, k=1, \ldots, N_{r}$ are assumed to be independent, the obtained PDF from [47, Theorem 1] deviates from the empirical results.

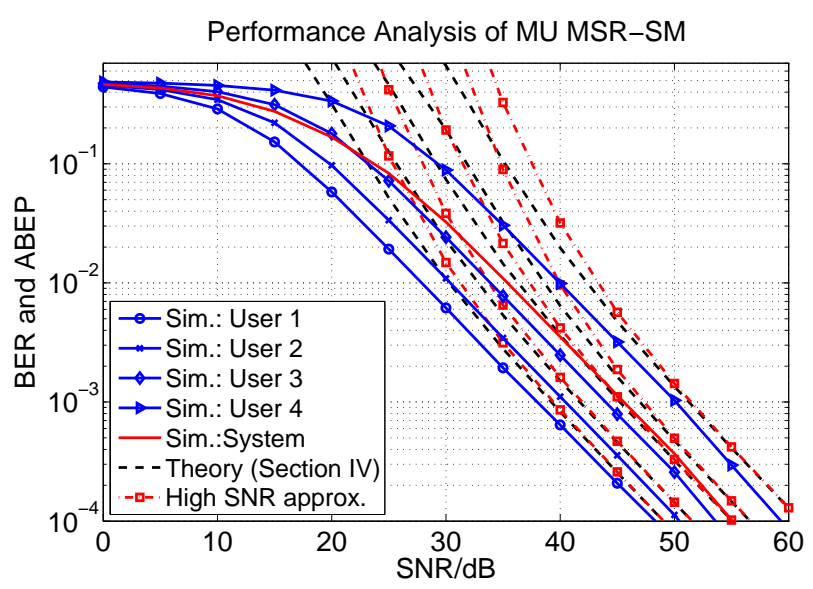

Fig. 3. Performance analysis of MU MSR-SM $\left(N_{s}=2\right)$ for four users, when ZF with P-CSIT is employed: simulation results vs. the bounds in Section IV. The high SNR approximation of the ABEP is calculated using the PEP given in (32). Setup: $N_{t}=16, N_{r}=4, \xi_{i}, i=1, \ldots, N_{u}$, takes the values 1 , $0.75,0.5$, and 0.25 for the user $1,2,3$, and 4 , respectively.

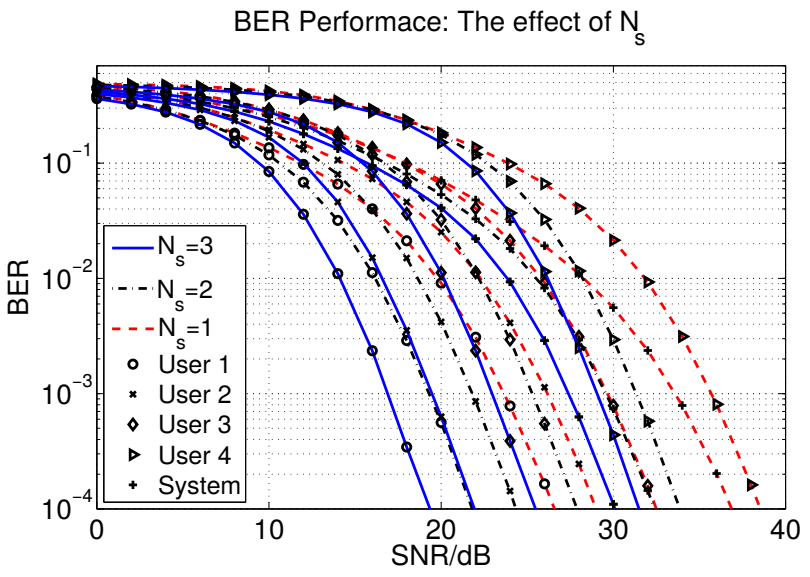

Fig. 4. BER performance of MU MSR-SM as a function of $N_{s}$. Setup: $N_{t}=20, N_{r}=4, N_{u}=4$. The spectral efficiency is $8 \mathrm{bpcu}$.

\section{B. Validation of the Theoretical Analysis}

The upper bounds derived in Section IV are compared against Monte Carlo simulations in Figs. 2 and 3. Furthermore, the same figures illustrate the upper bounds of the ABEP for the system and for each user when the high SNR approximation of the PEP in (32) is used. Note that Figs. 2 and 3 present the BER of the proposed architecture in very high SNRs solely for validating the theoretical framework of this paper. The inspection of Figs. 2 and 3 indicates that the analytical bounds of the ABEP are tight in the high SNR, both for (29) and (32). More specifically, in the high SNR, the analytical results can be considered as an excellent approximation of the simulation results. In contrast, in the low SNR, there is a difference between the theoretical and simulation results. However, this is a well known phenomenon that originates from using union bound methods [42]. Finally, the diversity order and coding gain analysis of Section $\mathrm{V}$ is also verified from Figs. 2 and 3. In more detail, the slope of the simulated BER curves of each user is $L_{\mathrm{MU}}=N_{t}-N_{u} N_{r}+1$. In addition, the simulated curves show that the behavior of the coding 


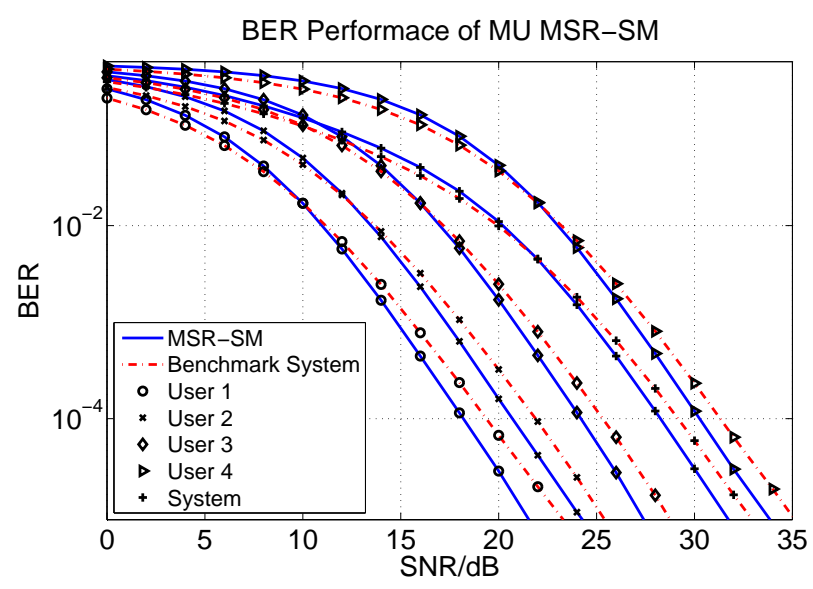

(a) The spectral efficiency is $2 \mathrm{bpcu}$, with $N_{s}=1$.

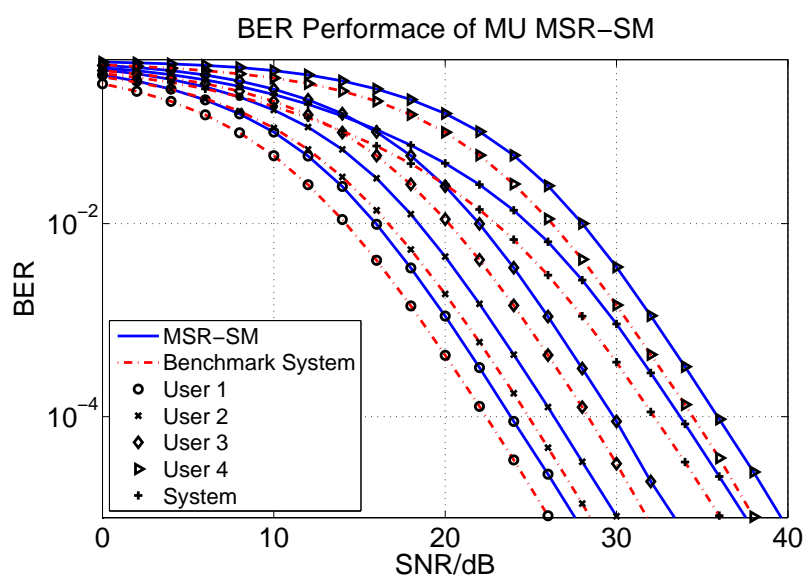

(b) The spectral efficiency is $4 \mathrm{bpcu}$, with $N_{s}=1$.

Fig. 5. BER performance of MU MSR-SM versus benchmark system (conventional MIMO broadcast channel with SMX). Setup: $N_{t}=10, N_{r}=2$, $N_{u}=4$.

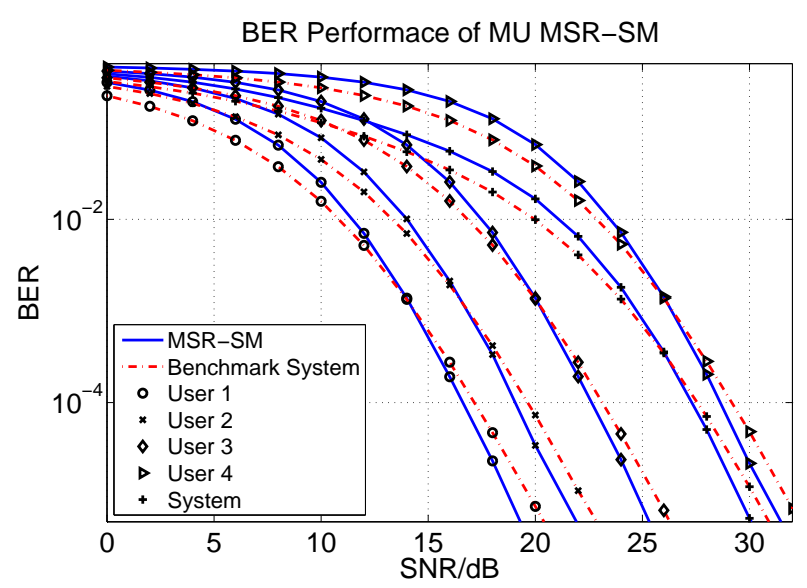

(a) The spectral efficiency is $4 \mathrm{bpcu}$, with $N_{s}=2$.

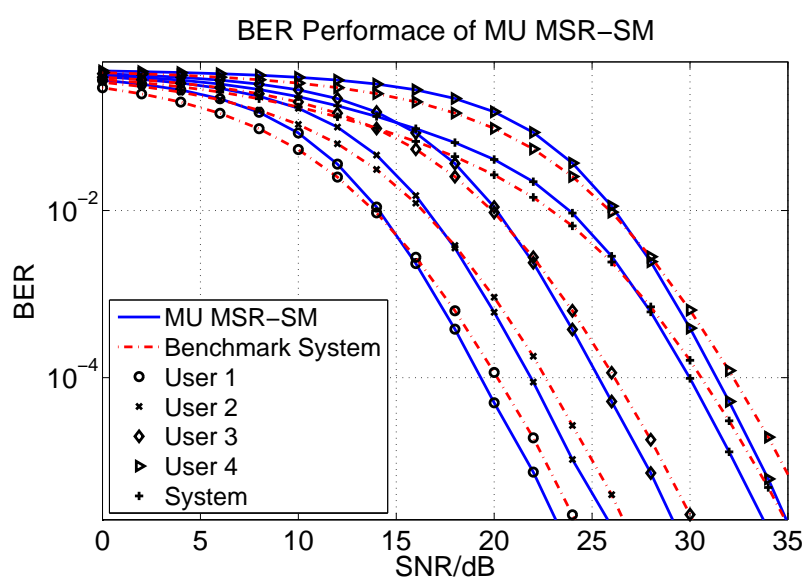

(b) The spectral efficiency is $8 \mathrm{bpcu}$, with $N_{s}=3$.

Fig. 6. BER performance of MU MSR-SM versus benchmark system (conventional MIMO broadcast channel with SMX). Setup: $N_{t}=20, N_{r}=4$, $N_{u}=4$.

gain of the $i$-th user depends on $\xi_{i}$. Similarly, the simulated curves show that the system coding gain is dominated by $\xi_{\min }$. These conclusions are in perfect agreement with the analysis presented in Section V.

\section{Analysis of the Impact of $N_{s}$}

Figure 4 shows the BER of MU MSR-SM as a function of $N_{s}$. It shows, in particular, that the BER of each user gets better as $N_{s}$ increases. This happens because higher values of $N_{s}$ require a lower modulation order of Quadrature Amplitude Modulation (QAM) in order to achieve the same spectral efficiency. Table I, however, shows that the optimal value of $N_{s}$ is not necessarily equal to $N_{r}$, i.e., the conventional MIMO broadcast channel.

\section{BER Comparison with the Conventional Broadcast Chan- nel}

Figures 5 and 6 show the BER of MU MSR-SM and of the conventional MIMO broadcast channel (benchmark system) for different system setups. As shown in Figs. 5(a), 6(a), and 6(b), in the low SNR, conventional MIMO offers a slightly better BER than the new scheme. In the high SNR, on the other hand, the new architecture outperforms the benchmark system. More specifically, in Figs. 5(a), 6(a), and 6(a), and at $\mathrm{BER}=10^{-4}$, MU MSR-SM provides a gain of $1.4,0.8$, and $1 \mathrm{~dB}$, respectively. In contrast, in Fig. 6(b), MU MSR-SM is outperformed by the benchmark system. However, even in this case, MU MSR-SM retains its complexity benefits at the transmitter. These findings are in agreement with the mathematical analysis of the ratio of the coding gains in (43).

\section{E. Energy Efficiency Comparison with the Conventional Broadcast Channel}

In this section, the energy efficiency of MU MSR-SM is studied using the Relative Average Energy Reduction (RAER) performance metric, which is defined as follows:

$$
\operatorname{RAER}[\%]=\left[1-10^{-\frac{\Delta_{\mathrm{SNR}}}{10}}\right] \times 100 \% .
$$


TABLE II

RAER OF MU MSR-SM WITH RESPECT TO THE CONVENTIONAL MIMO BROADCAST CHANNEL.

\begin{tabular}{c|c|c|c}
\hline \hline Configuration & $k_{\text {system }}(\mathrm{bpsp})$ & Target BER & RAER [\%] \\
\hline \hline$N_{t}=20, N_{r}=4, N_{u}=4$ & 32 & $10^{-1}$ & $-20 \%$ \\
$N_{t}=20, N_{r}=4, N_{u}=4$ & 32 & $10^{-2}$ & $1 \%$ \\
$N_{t}=20, N_{r}=4, N_{u}=4$ & 32 & $10^{-4}$ & $18 \%$ \\
\hline \hline
\end{tabular}

In (44), $\Delta_{\mathrm{SNR}}$ denotes the SNR difference (in $\mathrm{dB}$ ) between MU MSR-SM and the conventional MIMO broadcast channel for a given BER. It is worth mentioning that only the energy consumption for the RF power transmission is considered. Both architectures, in fact, have almost the same circuits energy consumption, since they employ the same number of RF front-ends.

Table II presents the system RAER of MU MSR-SM with respect to the conventional MIMO broadcast channel, by assuming the same system setup as in Fig. 6(a). Both schemes provide the same BER and the same spectral efficiency. If $\mathrm{BER}=10^{-1}$, the conventional MIMO broadcast channel is more energy efficient. For practical values of the BER less than $10^{-2}$, on the other hand, MU MSR-SM becomes more energy efficient. For example, an energy efficiency gain of $18 \%$ is achieved by MU MSR-SM at $\mathrm{BER}=10^{-4}$.

\section{CONCLUSIONS}

In this paper, the incorporation of MSR-SM for application to the MIMO broadcast channel is introduced and its BER performance is mathematically studied. More specifically, based on the union bound technique, an accurate mathematical framework for its performance evaluation is proposed and discussed. From this framework, it is proved that MSR-SM provides the same diversity order as the conventional MIMO broadcast channel, while offering a better coding gain in the high SNR regime. Also, this performance gain is achieved with a reduction of the complexity of the transmitter. Numerical simulations are shown in order to substantiate the gain predicted by the analysis. As a result, MSR-SM is shown to be a promising transmission scheme for the MIMO broadcast channel.

\section{REFERENCES}

[1] G. J. Foschini and M. J. Gans, "On limits of wireless communications in a fading environment when using multiple antennas," Wireless Personal Communications, vol. 6, no. 6, pp. 311-335, 1998.

[2] E. Telatar, "Capacity of multi-antenna gaussian channels," European Trans. on Telecommun., vol. 10, no. 6, pp. 585-595, Nov. / Dec. 1999.

[3] M. Di Renzo, H. Haas, A. Ghrayeb, S. Sugiura, and L. Hanzo, "Spatial modulation for generalized MIMO: Challenges, opportunities, and implementation," Proc. IEEE, vol. 102, no. 1, pp. 56-103, Jan 2014.

[4] M. Di Renzo, H. Haas, and P. M. Grant, "Spatial modulation for multiple-antenna wireless systems: A survey," IEEE Commun. Mag., vol. 49, no. 12, pp. 182-191, December 2011.

[5] P. Yang, M. Di Renzo, Y. Xiao, S. Li, and L. Hanzo, "Design guidelines for spatial modulation," IEEE Commun. Surveys Tut., vol. 17, no. 1, pp. 6-26, Firstquarter 2015.

[6] M. Di Renzo and H. Haas, "Bit error probability of SM-MIMO over generalized fading channels," IEEE Trans. on Veh. Technol., vol. 61, no. 3, pp. 1124-1144, March 2012.

[7] R. Mesleh, H. Haas, S. Sinanović, C. W. Ahn, and S. Yun, "Spatial modulation," IEEE Trans. on Veh. Tech., vol. 57, no. 4, pp. 2228 2241, Jul. 2008
[8] J. Jeganathan, A. Ghrayeb, and L. Szczecinski, "Spatial modulation: Optimal detection and performance analysis," IEEE Commun. Lett., vol. 12 , no. 8, pp. 545-547, 2008.

[9] A. Stavridis, S. Sinanović, M. D. Renzo., H. Haas, and P. Grant, "An energy saving base station employing spatial modulation," in IEEE 17th Int. Workshop on Computer Aided Modeling and Design of Communication Links and Networks (CAMAD), Sep. 17-19 2012, pp. $231-235$.

[10] A. Stavridis, S. Sinanović, M. D. Renzo., and H. Haas, "Energy evaluation of spatial modulation at a multi-antenna base station," in Proc. of the 78th IEEE Veh. Tech. Conf. (VTC), Las Vegas, USA, Sep. 2-5, 2013.

[11] J. Jeganathan, A. Ghrayeb, L. Szczecinski, and A. Ceron, "Space shift keying modulation for MIMO channelsmeh," IEEE Trans. on Wireless Commun., vol. 8, no. 7, pp. 3692-3703, Jul. 2009.

[12] J. Jeganathan, A. Ghrayeb, and L. Szczecinski, "Generalized Space Shift Keying Modulation for MIMO Channels," in Proc. IEEE 19th Intern. Symp. on Personal, Indoor and Mobile Radio Commun. PIMRC 2008, Cannes, France, 15-18 Sep. 2008, pp. 1-5.

[13] R. Mesleh, M. Di Renzo, H. Haas, and P. M. Grant, "Trellis coded spatial modulation," IEEE Trans. on Wireless Commun., vol. 9, no. 7, pp. 2349-2361, Jul. 2010.

[14] M. Raed, I. Salama, A. Hadi, and A. Mansour, "Performance analysis of space shift keying (SSK) modulation with multiple cooperative relays," EURASIP J. on Adv. in Signal Process., 2012.

[15] M. Wen, X. Cheng, H. Poor, and B. Jiao, "Use of SSK modulation in two-way amplify-and-forward relaying," IEEE Trans. on Veh. Techn. vol. 63, no. 3, pp. 1498-1504, March 2014.

[16] P. Som and A. Chockalingam, "BER analysis of space shift keying in cooperative multi-hop multi-branch DF relaying," in Proc. of the 78th IEEE Veh. Tech. Conf. (VTC), Las Vegas, USA, Sep. 2-5, 2013.

[17] — , "Performance analysis of space-shift keying in decode-andforward multihop MIMO networks," IEEE Trans. on Veh. Techn., vol. 64, no. 1, pp. 132-146, Jan 2015.

[18] M. Maleki, H. Bahrami, S. Beygi, M. Kafashan, and N. Tran, "Space modulation with CSI: Constellation design and performance evaluation,' IEEE Trans. on Veh. Technol., vol. 62, no. 4, pp. 1623-1634, May 2013.

[19] P. Yang, Y. Xiao, Y. Yu, and S. Li, "Adaptive spatial modulation for wireless MIMO transmission systems," IEEE Commun. Lett., vol. 15, no. 6, pp. 602-604, June 2011.

[20] P. Yang, Y. Xiao, Y. Yu, L. Li, Q. Tang, and S. Li, "Simplified adaptive spatial modulation for limited-feedback MIMO systems," IEEE Trans. on Veh. Technol., vol. 62, no. 6, pp. 2656-2666, July 2013.

[21] P. Yang, Y. Xiao, B. Zhang, S. Li, M. El-Hajjar, and L. Hanzo, "Power allocation-aided spatial modulation for limited-feedback MIMO systems," IEEE Trans. on Veh. Technol., vol. 64, no. 5, pp. 2198-2204, May 2015.

[22] M. Veedu, C. Murthy, and L. Hanzo, "Single-RF spatial modulation relying on finite-rate phase-only feedback: Design and analysis," IEEE Trans. on Veh. Technol., vol. PP, no. 99, pp. 1-1, 2015.

[23] A. Younis, R. Mesleh, and H. Haas, "Quadrature spatial modulation performance over Nakagami-m fading channels," IEEE Trans.on Veh. Technol., vol. PP, no. 99, pp. 1-1, 2015.

[24] S. Sugiura, S. Chen, and L. Hanzo, "Generalized space-time shift keying designed for flexible diversity-, multiplexing- and complexity-tradeoffs," IEEE Trans. on Wireless Commun., vol. 10, no. 4, pp. 1144 -1153, Apr. 2011.

[25] N. Serafimovski, A. Younis, R. Mesleh, P. Chambers, M. D. Renzo, C.-X. Wang, P. M. Grant, M. A. Beach, and H. Haas, "Practical implementation of spatial modulation," IEEE Trans. on Veh. Tech., vol. 62, no. 9, pp. 4511-4523, 2013.

[26] A. Younis, W. Thompson, M. D. Renzo, C.-X. Wang, M. A. Beach, H. Haas, and P. M. Grant, "Performance of spatial modulation using measured real-world channels," in Proc. of the 78th IEEE Veh. Tech. Conf. (VTC), Las Vegas, USA, Sep. 2-5 2013.

[27] J. Zhang, Y. Wang, L. Ding, and N. Zhang, "Bit error probability of spatial modulation over measured indoor channels," IEEE Trans. on Wireless Commun., vol. 13, no. 3, pp. 1380-1387, March 2014.

[28] L.-L. Yang, "Transmitter preprocessing aided spatial modulation for multiple-input multiple-output systems," in Proc. of 73rd IEEE Veh.Techn. Conf. (VTC Spring), May 2011, pp. 1 -5.

[29] R. Zhang, L.-L. Yang, and L. Hanzo, "Generalised pre-coding aided spatial modulation," IEEE Trans. on Wireless Commun., vol. 12, no. 11, pp. 5434-5443, November 2013.

[30] - "Error probability and capacity analysis of generalised pre-coding aided spatial modulation," IEEE Trans. on Wireless Commun., vol. PP, no. 99, pp. 1-1, 2014. 
[31] A. Stavridis, S. Sinanović, M. Di Renzo, and H. Haas, "Transmit precoding for receive spatial modulation using imperfect channel knowledge," in Proc. of 75th IEEE Veh. Techn. Conf. (VTC Spring), 2012.

[32] A. Stavridis, D. Basnayaka, M. Di Renzo, and H. Haas, "Average bit error probability of receive-spatial modulation using zero-forcing precoding," in IEEE 19th Int. Workshop on Computer Aided Modeling and Des. of Commun. Links and Netw. (CAMAD), 2014.

[33] A. Stavridis, S. Sinanović, M. D. Renzo, and H. Haas, "A power saving dual-hop architecture based on hybrid spatial modulation," in 2012 Conf. Record of the Forty Sixth Asilomar Conf. on Signals, Systems and Computers (ASILOMAR), Nov. 4-7 2012, pp. 1366-1370.

[34] A. Stavridis, D. Basnayaka, S. Sinanovic, M. Di Renzo, and H. Haas, "A virtual MIMO dual-hop architecture based on hybrid spatial modulation," IEEE Trans. on Commun., vol. 62, no. 9, pp. 3161-3179, Sept 2014.

[35] C. Masouros and L. Hanzo, "Dual layered MIMO transmission for increased bandwidth efficiency," IEEE Trans. on Veh. Technol., vol. PP, no. 99, pp. 1-1, 2015.

[36] R. Zhang, L.-L. Yang, and L. Hanzo, "Energy pattern aided simultaneous wireless information and power transfer," IEEE J. on Sel. Areas in Commun., vol. 33, no. 8, pp. 1492-1504, Aug 2015.

[37] M. Kountouris and J. Andrews, "Downlink SDMA with limited feedback in interference-limited wireless networks," IEEE Trans. on Wireless Commun., vol. 11, no. 8, pp. 2730-2741, August 2012.

[38] M. Matthaiou, N. Chatzidiamantis, G. Karagiannidis, and J. Nossek, "ZF detectors over correlated K fading MIMO channels," IEEE Trans. on Commun,., vol. 59, no. 6, pp. 1591-1603, June 2011.

[39] S. Narayanan, M. J. Chaudhary, A. Stavridis, R. Di Renzo, F. Graziosi, and H. Haas, "Multi-user spatial modulation MIMO," in Proc. of IEEE Wireless Communications and Networking Conference (WCNC), Apr. 6-9, 2014.

[40] X. Li, Y. Zhang, L. Xiao, X. Xu, and J. Wang, "A novel precoding scheme for downlink multi-user spatial modulation system," in Proc. IEEE 24th Int. Symp. Pers. Indoor and Mobile Radio Commun. (PIMRC), Sept 2013, pp. 1361-1365.

[41] A. Wiesel, Y. Eldar, and S. Shamai, "Zero-Forcing Precoding and Generalized Inverses," IEEE Trans. on Signal Process., vol. 56, no. 9, pp. $4409-4418$, Sept. 2008.

[42] J. G. Proakis, Digital Communications, 4th ed. New York, NY, USA: McGraw-Hill, 2000.

[43] M. Chiani, D. Dardari, and M. K. Simon, "New exponential bounds and approximations for the computation of error probability in fading channels," IEEE Trans. on Wireless Commun., vol. 2, no. 4, pp. 840-845, July 2003.

[44] X. Shao, J. Yuan, and Y. Shao, "Error performance analysis of linear zero forcing and MMSE precoders for MIMO broadcast channels," IET Commun., vol. 1, no. 5, pp. 1067-1074, Oct 2007.

[45] C.-J. Chen and L.-C. Wang, "Performance analysis of scheduling in multiuser MIMO systems with zero-forcing receivers," IEEE J. Sel. Areas Commun., vol. 25, no. 7, pp. 1435-1445, September 2007.

[46] D. Gore, R. Heath, and A. Paulraj, "Transmit selection in spatial multiplexing systems," IEEE Commun. Lett., vol. 6, no. 11, pp. 491-493, Nov 2002.

[47] M.-S. Alouini, A. Abdi, and M. Kaveh, "Sum of gamma variates and performance of wireless communication systems over Nakagami-fading channels," IEEE Trans. on Veh. Technol., vol. 50, no. 6, pp. 1471-1480, Nov 2001.

[48] I. S. Gradshteyn and I. M. Ryzhik, Table of Integrals, Series, and Products, 7th ed., A. Jeffrey and D. Zwillinger, Eds. Academic Press, Mar. 2007, ISBN-10: 0123736374.

[49] Z. Wang and G. Giannakis, "A simple and general parameterization quantifying performance in fading channels," IEEE Trans. on Commun., vol. 51, no. 8, pp. 1389-1398, Aug 2003.

[50] A. Mehana and A. Nosratinia, "Diversity of MIMO linear precoding," IEEE Trans. Inf. Theory, vol. 60, no. 2, pp. 1019-1038, Feb 2014.

[51] W. Guan and K. Liu, "Diversity analysis of analog network coding with multi-user interferences," IEEE Trans. Wireless Commun., vol. 12, no. 2, pp. 668-679, February 2013.

[52] H. Dhillon, R. Ganti, F. Baccelli, and J. Andrews, "Modeling and analysis of k-tier downlink heterogeneous cellular networks," IEEE Journal on Selected Areas in Communications, vol. 30, no. 3, pp. 550 -560 , april 2012.

[53] M. Di Renzo and W. Lu, "Stochastic geometry modeling and performance evaluation of MIMO cellular networks using the equivalent-indistribution (EiD)-based approach," EEE Trans. on Commun., vol. 63, no. 3, pp. 977-996, March 2015.
[54] K. Ntontin, M. Di Renzo, A. Perez-Neira, and C. Verikoukis, "Analog network coding in the multiple access relay channel: Error rate analysis and optimal power allocation," IEEE Trans. on Wireless Commun., vol. 14, no. 6, pp. 3015-3032, June 2015. 